\title{
Production of ent-kaurene from lignocellulosic hydrolysate in Rhodosporidium toruloides
}

\author{
Gina M. Geiselman ${ }^{1,2 \dagger}$, Xun Zhuang ${ }^{1,2 \dagger}$, James Kirby ${ }^{1,2}$, Mary B. Tran-Gyamfi, ${ }^{1,}$, Jan-Philip Prahl1,4, \\ Eric R. Sundstrom ${ }^{3,4}$, Yuqian Gao5, Nathalie Munoz Munoz ${ }^{5}$, Carrie D. Nicora ${ }^{5}$, Derek M. Clay ${ }^{1,2}$, Gabriella Papa ${ }^{3,4}$, \\ Kristin E. Burnum-Johnson ${ }^{5}$, Jon K. Magnuson ${ }^{6}$, Deepti Tanjore ${ }^{3,4}$, Jeffrey M. Skerker ${ }^{7}$ and John M. Gladden ${ }^{1,2^{*}}$ (D)
}

\begin{abstract}
Background: Rhodosporidium toruloides has emerged as a promising host for the production of bioproducts from lignocellulose, in part due to its ability to grow on lignocellulosic feedstocks, tolerate growth inhibitors, and co-utilize sugars and lignin-derived monomers. Ent-kaurene derivatives have a diverse range of potential applications from therapeutics to novel resin-based materials.

Results: The Design, Build, Test, and Learn (DBTL) approach was employed to engineer production of the non-native diterpene ent-kaurene in $R$. toruloides. Following expression of kaurene synthase (KS) in $R$. toruloides in the first DBTL cycle, a key limitation appeared to be the availability of the diterpene precursor, geranylgeranyl diphosphate (GGPP). Further DBTL cycles were carried out to select an optimal GGPP synthase and to balance its expression with KS, requiring two of the strongest promoters in R. toruloides, ANT (adenine nucleotide translocase) and TEF1 (translational elongation factor 1) to drive expression of the KS from Gibberella fujikuroi and a mutant version of an FPP synthase from Gallus gallus that produces GGPP. Scale-up of cultivation in a $2 \mathrm{~L}$ bioreactor using a corn stover hydrolysate resulted in an ent-kaurene titer of $1.4 \mathrm{~g} / \mathrm{L}$.
\end{abstract}

Conclusion: This study builds upon previous work demonstrating the potential of $R$. toruloides as a robust and versatile host for the production of both mono- and sesquiterpenes, and is the first demonstration of the production of a non-native diterpene in this organism.

Keywords: Rhodotorula, Mevalonate pathway, Diterpene, Geranylgeranyl pyrophosphate synthase, Mutant farnesyl pyrophosphate synthase, Metabolic engineering

\section{Background}

The diverse terpenoid family contains over 70,000 unique compounds that are predominantly produced from two fundamental isoprenoid building blocks, the C5 prenyl phosphates, dimethylallyl diphosphate (DMAPP) and

\footnotetext{
*Correspondence: jmgladden@lbl.gov

${ }^{\dagger}$ Gina M. Geiselman and Xun Zhuang contributed equally to this work

2 Department of Biomass Science and Conversion Technology, Sandia National Laboratories, 7011 East Ave, Livermore, CA 94550, USA

Full list of author information is available at the end of the article
}

isopentenyl diphosphate (IPP), via the mevalonate (MVA) pathway or the 1-deoxyxylulose 5-phosphate (DXP) pathway [1-3]. These precursors are combined to generate longer prenyl phosphates such as the $\mathrm{C} 10$ monoterpene precursor, geranyl diphosphate (GPP), the C15 sesquiterpene precursor, farnesyl diphosphate (FPP), and C20 diterpene precursor, geranylgeranyl diphosphate (GGPP) $[4,5]$.

Diterpenes are less volatile than monoterpenes and sesquiterpenes, and have various industrial and biological applications from resin-based adhesives to potential 
new drugs [6-8]. In plants, diterpenes play diverse roles such as protection against pathogens and herbivores [9] and production of growth regulators such as gibberellins [10-12]. Plants, fungi and bacteria produce gibberellins from a universal precursor, ent-kaurene, which may be synthesized from GGPP in a single step or in two steps, via the intermediate ent-copalyl diphosphate (CDP) [13]. Gibberellins are used as a sustainable means of increasing yields and stress-tolerance in agriculture and floriculture but are currently expensive, leading to calls for biotechnological approaches to reduce cost and to increase the diversity of commercially available gibberellins [13, 14]. Besides serving as the precursor to gibberellins, ent-kaurene may also be transformed into a variety of bioactive derivatives, with a range of potential applications. Anti-microbial, anti-cancer, and anti-inflammatory activities are among the properties ascribed to kaurene-derived diterpenoids, such as ent-kaur-16-en-19-oic acid (kaurenoic acid) [15]. In traditional Chinese medicine, pharmacologically active ent-kaurene diterpenoids from Isodon plants (Lamiaceae) are used to treat inflammation and cancers [16]. Finally, kaurene-derivatives may play a key role in the development of new renewable polymers [17].

Only a few studies have reported on engineering microbes to produce ent-kaurene. For example, kaurene synthase (KS) from Gibberella fujikuroi was expressed in Aspergillus nidulans and although ent-kaurene production was demonstrated, it was not quantified [18]. In another study, CDP synthase and KS from Stevia rebaudiana were expressed in Escherichia coli along with three genes from the native DXP pathway, yielding $578 \mathrm{mg} / \mathrm{L}$ ent-kaurene from a glycerol-based $(20 \mathrm{~g} / \mathrm{L})$ medium in a $1 \mathrm{~L}$ bioreactor [19]. To develop a truly sustainable solution for microbial production of bioproducts, the next step is to transition preliminary findings from studies like these to microbial hosts capable of utilizing cheap renewable carbon sources that do not compete with our food supply, such as lignocellulose.

Lignocellulose poses a challenge in that it is difficult to deconstruct into fermentable carbon, and many deconstruction technologies can produce toxic molecules that inhibit microbial growth and productivity. Much work has been done to develop technologies for efficient lignocellulose deconstruction and generation of non-toxic hydrolysates that are easily converted into bioproducts by microbial hosts capable of consuming lignocellulosic sugars. For example, a process was recently developed that generates clean lignocellulosic hydrolysates, called DMR-EH, with low concentrations of microbial growth inhibitors such as acetate, furfural, and 5-hydroxymethylfurfural and up to $230 \mathrm{~g} / \mathrm{L}$ of monomeric sugars [20, 21].
This hydrolysate has been used to produce other terpene bioproducts, such as 1,8-cineole in a robust microbial host, Rhodosporidium toruloides [22].

Rhodosporidium toruloides, has emerged as a promising host for the utilization of lignocellulosic feedstocks, in part because it can withstand osmotic stress [23] and potential growth inhibitors found in biomass hydrolysates [24]. In addition, $R$. toruloides adapts well to the mixed carbon sources in low-cost lignocellulosic feedstocks, utilizing mixtures of C5 and C6 sugars in combination with lignin-derived compounds such as p-coumarate $[25,26] . R$. toruloides can be grown to high cell densities, surpassing $150 \mathrm{~g} / \mathrm{L}$ dry cell weight in highgravity fermentation [27]. To date, $R$. toruloides has been engineered to produce a number of bioproducts, including lipids, the terpene biofuel candidates 1,8-cineole and bisabolene, and the non-ribosomal peptide, indigoidine $[22,26,28,29]$. This work explores the potential for $R$. toruloides to produce diterpene products from lignocellulosic biomass, targeting the production of ent-kaurene from corn stover DMR-EH hydrolysate.

\section{Results}

To produce ent-kaurene in $R$. toruloides, we selected kaurene synthase from Gibberella fujikuroi (GfKS) [30, 31] because it generates ent-kaurene directly from GGPP (Fig. 1) [13]. In plant and bacterial systems, ent-kaurene is synthesized from GGPP in a two-step process, via CDP, while G. fujikuroi and other fungi contain bifunctional $\mathrm{CDP} / \mathrm{KS}$ enzymes that generate ent-kaurene directly from GGPP (Fig. 1) [13]. The native promoters GAPDH (glyceraldehyde 3-phosphate dehydrogenase) and ANT (adenine nucleotide translocase) were chosen for heterologous expression of GfKS based on analysis of RNAseq data from a previous study that suggests that they both are constitutive and drive a high level of gene expression [32].

Constructs were transformed into $R$. toruloides by Agrobacterium tumefaciens mediated transformation (ATMT), which results in random chromosomal integration of transgenes. Typically, 40 transformants were selected for initial measurement of ent-kaurene production and then the three highest-titer strains for each construct were compared in triplicate. Following screening of strains transformed with constructs harboring $\mathrm{P}_{A N T}$-GfKS (construct 1) and $\mathrm{P}_{\text {GAPDH }}$-GfKS (construct 2 ), we found that similar maximum ent-kaurene titers of $15-20 \mathrm{mg} / \mathrm{L}$ were reached in $\mathrm{YPD}_{10}$ medium (YPD containing $100 \mathrm{~g} / \mathrm{L}$ glucose; Fig. 2; constructs are listed in Table 1). The low ent-kaurene titers observed relative to other heterologous terpenes produced in this organism (typically several hundred $\mathrm{mg} / \mathrm{L}$ ) suggests that the availability of the GfKS substrate, GGPP, may be limiting. 


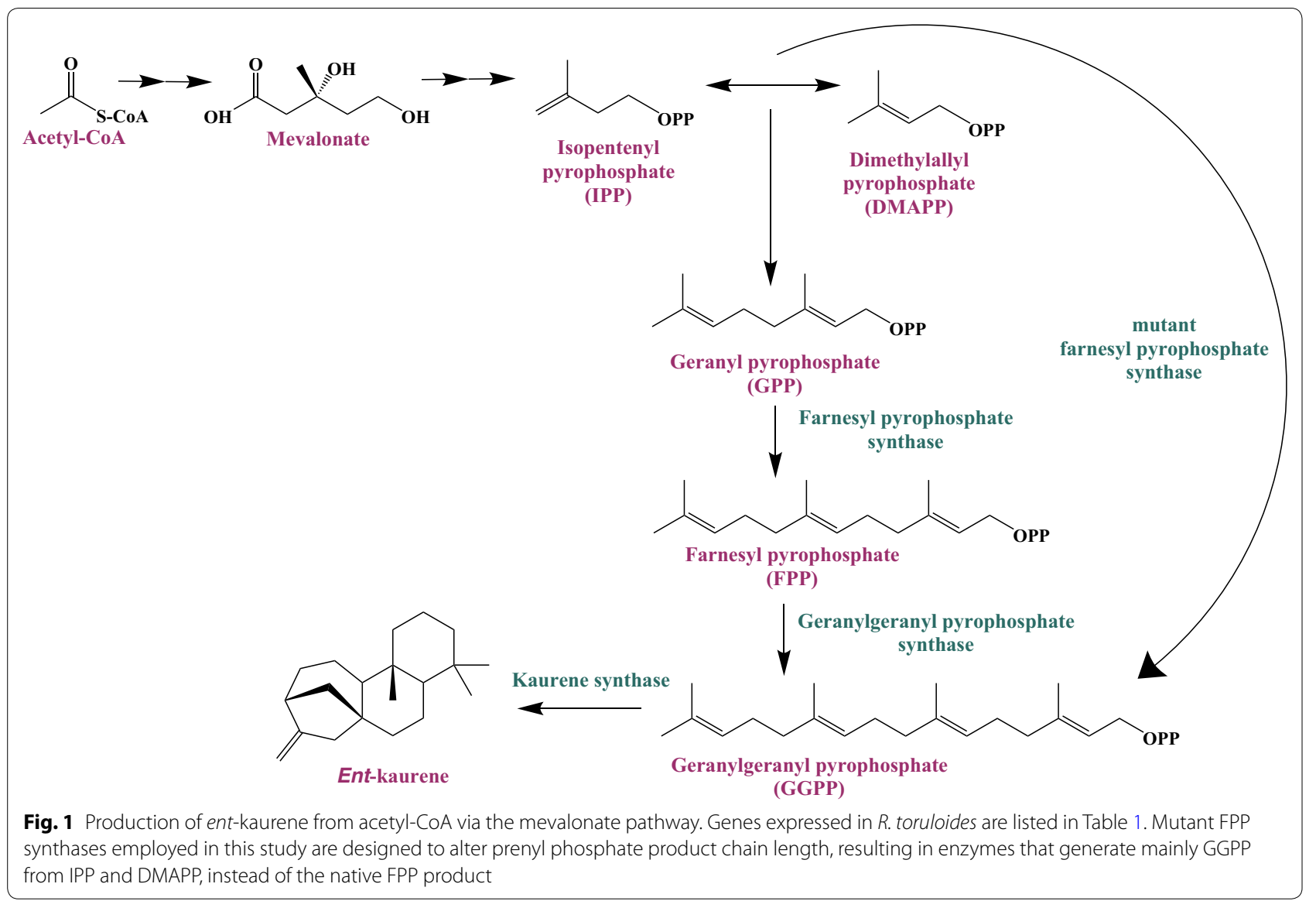

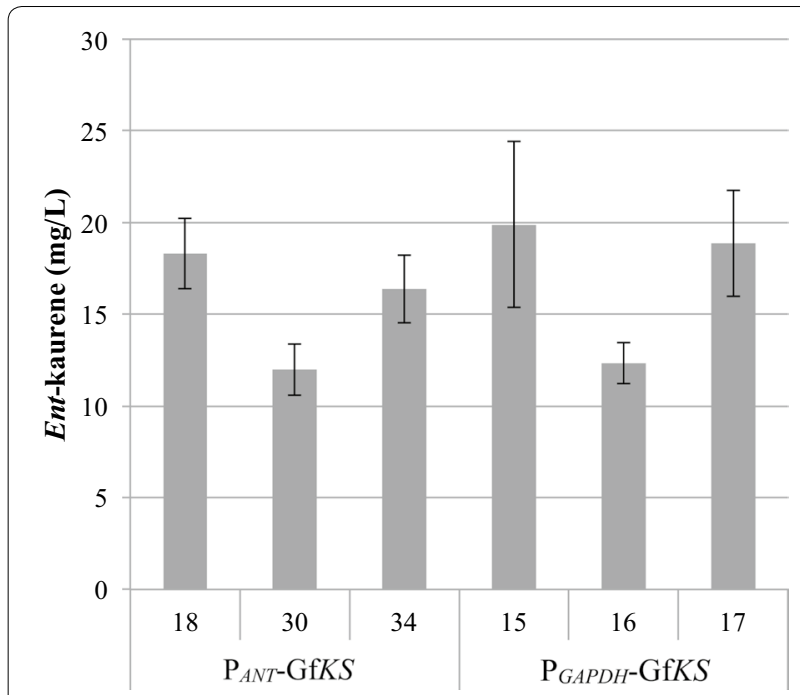

Fig. 2 Expression of kaurene synthase from Gibberella fujikuroi (GfKS) in $R$. toruloides. Ent-kaurene titer at 9 days is shown for the three highest-titer strains transformed with constructs $1\left(P_{\text {ANT }}\right.$-GFKS) and 2 $\left(\mathrm{P}_{\text {GAPDH }}-\mathrm{GFKS}\right)$. $(\mathrm{N}=3$, data shown as average \pm standard deviation, from a single experiment in $\mathrm{YPD}_{10}$ medium. ABFPUB identification numbers are listed in Table 1.)
Although $R$. toruloides produces carotenoids, they are produced at relatively low levels, indicating that native carbon flux to GGPP may be low [26].

Similar maximum titers were reached following transformation of $R$. toruloides with the $\mathrm{P}_{A N T} \mathrm{GfKS}$ and $\mathrm{P}_{\text {GAPDH }}$-GfKS constructs, even though the ANT promoter is natively stronger, as indicated by $A N T$ transcript levels and reporter studies [32]. To test whether this relative difference in promoter strength also applies to expression of the heterologous KS, GfKS copy number, transcript levels, and protein levels were measured for selected GfKS strains (Fig. 3). In strains harboring GfKS at similar copy numbers, GfKS transcript and protein levels were higher when expressed from $\mathrm{P}_{A N T}$ (strains ABFPUB_18 and 30) than from $\mathrm{P}_{\text {GAPDH }}$ (strain ABFPUB_16). In one $\mathrm{P}_{\text {GAPDH }}-\mathrm{Gf} K S$ strain, ABFPUB_15, GfKS transcript and protein levels are higher, but this is likely due to the twoto threefold higher GfKS copy number in ABFPUB_15.

Comparison of transcript and protein levels of KS with ent-kaurene titers can also give an indication of whether gene expression or metabolite pools are limiting ent-kaurene production. The $\mathrm{P}_{\text {GAPDH }}-\mathrm{GfKS}$ strain ABFPUB_16 had the lowest transcript and protein levels of the strains 
Table 1 Overview of constructs used to engineer ent-kaurene production in $R$. toruloides

\begin{tabular}{|c|c|c|}
\hline Plasmids & Genotypes/features & ABFPUB registry ID \\
\hline Construct 1 & $P_{A N T}-G F K S-H Y G^{R}$ & ABFPUB_1 \\
\hline Construct 2 & $P_{G A P D H}-G F K S-N A T^{R}$ & ABFPUB_3 \\
\hline Construct 3 & $\mathrm{P}_{\text {TEF } 1}-\mathrm{RtERG20(F91C)-KanMX^{R }}$ & ABFPUB_6 \\
\hline Construct 4 & $\mathrm{P}_{\text {TEF }}$-TCGGPPS(S239C,G29D)-KanMX $^{R}$ & ABFPUB_7 \\
\hline Construct 5 & $P_{T E F 1}-G g F P S(F 112 A)-K a n M X^{R}$ & ABFPUB_8 \\
\hline Construct 6 & $\mathrm{P}_{\text {TEF }}$ - RtBTS1-KanMX $X^{\mathrm{R}}$ & ABFPUB_9 \\
\hline Construct 7 & 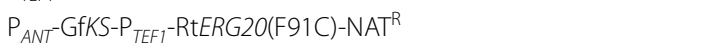 & ABFPUB_10 \\
\hline Construct 8 & $P_{A N T}-G F K S-P_{T E F 1}-T_{C} G G P P S\left(S 239 C\right.$, G295D)-NAT ${ }^{R}$ & ABFPUB_11 \\
\hline Construct 9 & $P_{A N T}-G f K S-P_{T E F T}-G g F P S(F 112 A)-N A T^{R}$ & ABFPUB_12 \\
\hline Construct 10 & 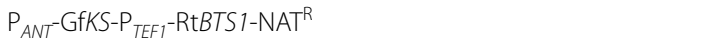 & ABFPUB_13 \\
\hline \multirow[t]{11}{*}{ Strains } & IFO0880 (WT) & ABFPUB_14 \\
\hline & IFO0880/P GAPDH $^{-G f K S-N A T^{R}}$ & ABFPUB_15-17 \\
\hline & IFO0880/P ${ }_{A N T}-G f K S-H Y G^{R}$ & ABFPUB_18, 30, 34 \\
\hline & IFO0880/P GAPDH $^{-G F K S-N A T^{R} / P_{\text {TEF } 1}-\text { RtERG20(F91C)-KanMX }}{ }^{R}$ & ABFPUB_47-49 \\
\hline & 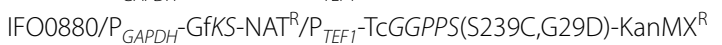 & ABFPUB_19, 20, 31 \\
\hline & 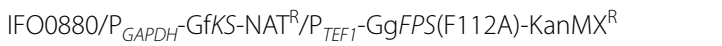 & ABFPUB_32, 33, 35 \\
\hline & 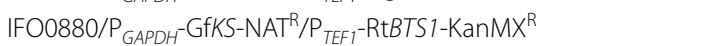 & ABFPUB_38-40 \\
\hline & IFO0880/P ANT $-G_{\text {GKS }}-\mathrm{P}_{\text {TEF }}-$ RtERG20(F91C)-NAT ${ }^{\mathrm{R}}$ & ABFPUB_41-43 \\
\hline & IFO0880/P ANT GfKS-P & ABFPUB_21, 22, 29 \\
\hline & IFO0880/P ${ }_{A N T}-G f K S-P_{T E F 1}-G g F P S(F 112 A)-N A T^{R}$ & ABFPUB_23, 26, 36 \\
\hline & IFO0880/P ${ }_{\text {ANT }}$-GfKS-P TEF $1-$ RtBTS1-NAT ${ }^{R}$ & ABFPUB_44-46 \\
\hline
\end{tabular}

Strains and plasmids used in this study are available upon request through the Agile BioFoundry Strain Registry (http://public-registry.agilebiofoundry.org// [37]) GAPDH glyceraldehyde 3-phosphate dehydrogenase, ANT, adenine nucleotide translocase, TEF1 translational elongation factor, GfKS, Gibberella fujikuroi kaurene synthase (NCBI Accession Number, Q9UVY5), RtERG20(F91C) mutant farnesyl pyrophosphate synthase from R. toruloides (PRQ75922.1), TcGGPPS(S239C,G295D) mutant geranylgeranyl pyrophosphate synthase from Taxus canadensis (AAD16018), GgFPS(F112A) mutant farnesyl pyrophosphate synthase from Gallus gallus (P08836.2) and RtBTS1 geranylgeranyl pyrophosphate synthase BTS1 from R. toruloides (PRQ72579)

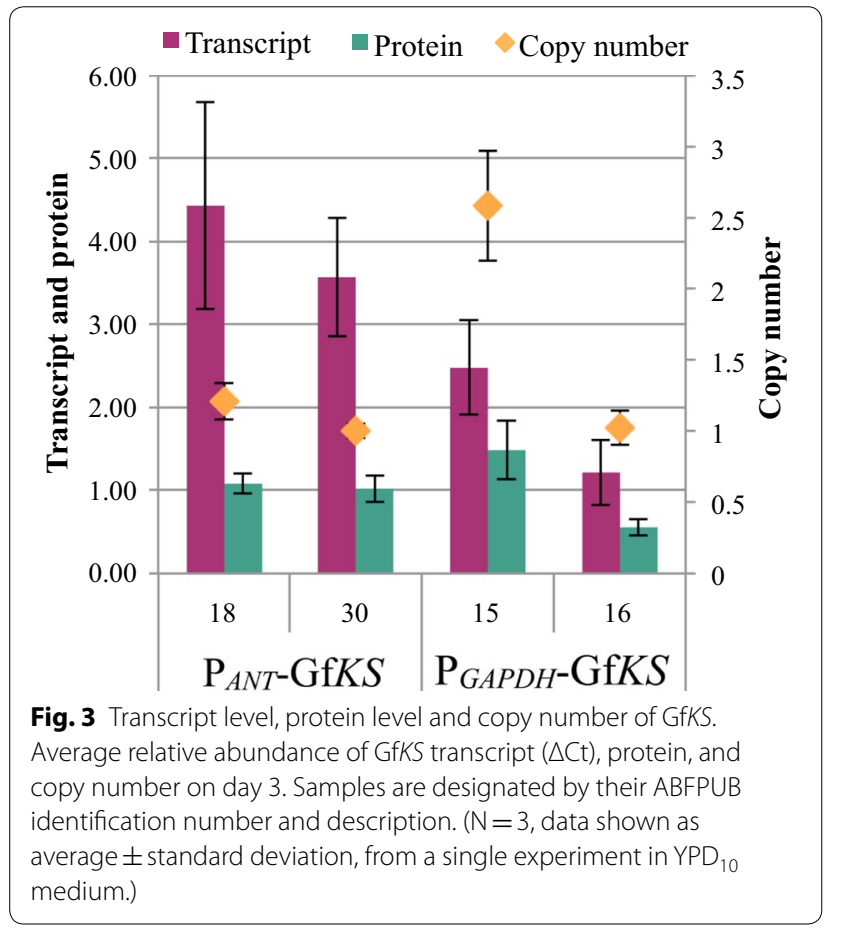

examined. While other strains (e.g., the $\mathrm{P}_{A N T}$-GfKS strain ABFPUB_30) had two- to fourfold higher transcript and protein levels, no substantial improvement in ent-kaurene titers was observed (Figs. 2 and 3). This indicates that a further increase in KS expression is not likely to significantly improve ent-kaurene production.

To investigate the possibility that GGPP levels may be a major factor limiting ent-kaurene titers, several GGPP synthases (GGPPSs) were selected for expression in a strain harboring GfKS. ABFPUB_16 was selected as a base strain, as it ranked most consistently as a low-variance, top-titer strain among the $80 \mathrm{Gf} K S$ transformants assayed in repeated screening trials. The native promoter TEF1 (translational elongation factor 1) was chosen to express each of four candidate GGPPSs: the native $R$. toruloides GGPPS (construct 6, $\mathrm{P}_{T E F 1}$ - $\mathrm{RtBTS1}$ ); a mutant of the $R$. toruloides farnesyl pyrophosphate (FPP) synthase (construct 3, $\mathrm{P}_{\text {TEF1 }}$-RtERG20(F91C)); a mutant GGPPS from Taxus canadensis (construct 4, $\mathrm{P}_{T E F-}$-TcGGPPS(S239C, G295D)); and a mutant FPP synthase from Gallus gallus (construct 5, $\mathrm{P}_{T E F 1}$-GgFPS(F112A)). The mutations, F91C and F112A, introduced to the R. toruloides and G. gallus 
FPP synthases, respectively, are designed to alter prenyl phosphate product chain length, resulting in enzymes that predominantly generate GGPP instead of the native FPP product [33]. The mutations S239C and G295D were previously identified in a carotenoid-based screen for improvements to the T. canadensis GGPPS [34]. Overexpression of RtERG2O(F91C) (construct 3) generally resulted in titers that were lower than or, at best, matching those of the parent strain ABFPUB_16, perhaps due to an unfavorable balance between FPP and GGPP biosynthesis, unintended enzyme inactivation due to mutation of the F91 residue, or feedback regulation of the native RtERG2O gene in $R$. toruloides (Fig. 4). However, overexpression of either the native $R$. toruloides GGPPS (construct 6) or the mutant GGPPS from T. canadensis (construct 4) resulted in more than two- and threefold increases in ent-kaurene titer, respectively. The most successful strategy was overexpression of the mutant FPP synthase from G. gallus (construct 5), which yielded a 17 -fold increase in ent-kaurene titer, to $345 \mathrm{mg} / \mathrm{L}$, in $\mathrm{YPD}_{10}$ medium. These results indicate that overexpression of GGPPS increases the available pool of GGPP for $\mathrm{KS}$, resulting in an improvement in ent-kaurene titers.

Optimizing carbon flux through a biosynthetic pathway requires balancing the expression of each pathway enzyme. The overexpression of GGPPS alongside KS shifted the balance of the pathway and resulted in an increase in ent-kaurene titers. To test whether this shift in pathway balance has caused KS to become a limiting factor, designs were made to balance KS and GGPPS expression by incorporating each $\mathrm{P}_{T E F T^{-}}$GGPPS and
$\mathrm{P}_{A N T}$-GfKS in a 1:1 ratio in the same construct. $\mathrm{P}_{A N T}$ was chosen over $\mathrm{P}_{G A P D H}$ in an attempt to restore any potential deficit in KS expression in the stacked KS GGPPS strains. After transformation into WT $R$. toruloides by ATMT, relative performance of the four GGPPSs was observed to remain the same but higher absolute ent-kaurene titers were achieved with GfKS expression under control of $\mathrm{P}_{A N T}$, suggesting that the KS may have become limiting as GGPP supply increased (Fig. 5). Co-transformation of $\mathrm{P}_{A N T}$ GfKS with $\mathrm{P}_{T E F-}$-TcGGPPS(S239C, G295D) (construct 8) and $\mathrm{P}_{T E F 1}-\mathrm{Gg} F P S(\mathrm{~F} 112 \mathrm{~A})$ (construct 9), resulted in ent-kaurene titers of 184 and $531 \mathrm{mg} / \mathrm{L}$ in $\mathrm{YPD}_{10}$ medium, respectively.

In order to understand how co-expression of KS and GGPPS in a single construct affected expression of each of these enzymes relative to iteratively stacking individual constructs into $R$. toruloides, transgene copy number, transcript and protein abundances of GfKS and GgFPS(F112A) were compared in three high-titer strains (Fig. 6). Transgene copy number was three- to fourfold higher in strains ABFPUB_23 and ABFPUB_26, which were generated by transformation of a single construct, relative to strain ABFPUB_35, which was constructed by gene stacking-transformation of ABFPUB_16 with

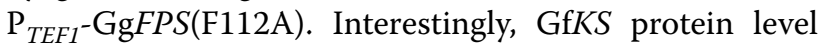
is similar in all three strains, while and $\operatorname{Gg} F P S(\mathrm{~F} 112 \mathrm{~A})$ protein level is higher in strains ABFPUB_23 and ABFPUB_26 than in strain ABFPUB_35. Overall, the highest average ent-kaurene titer was achieved in strain ABFPUB_26, which had the highest GgFPS(F112A) protein level.

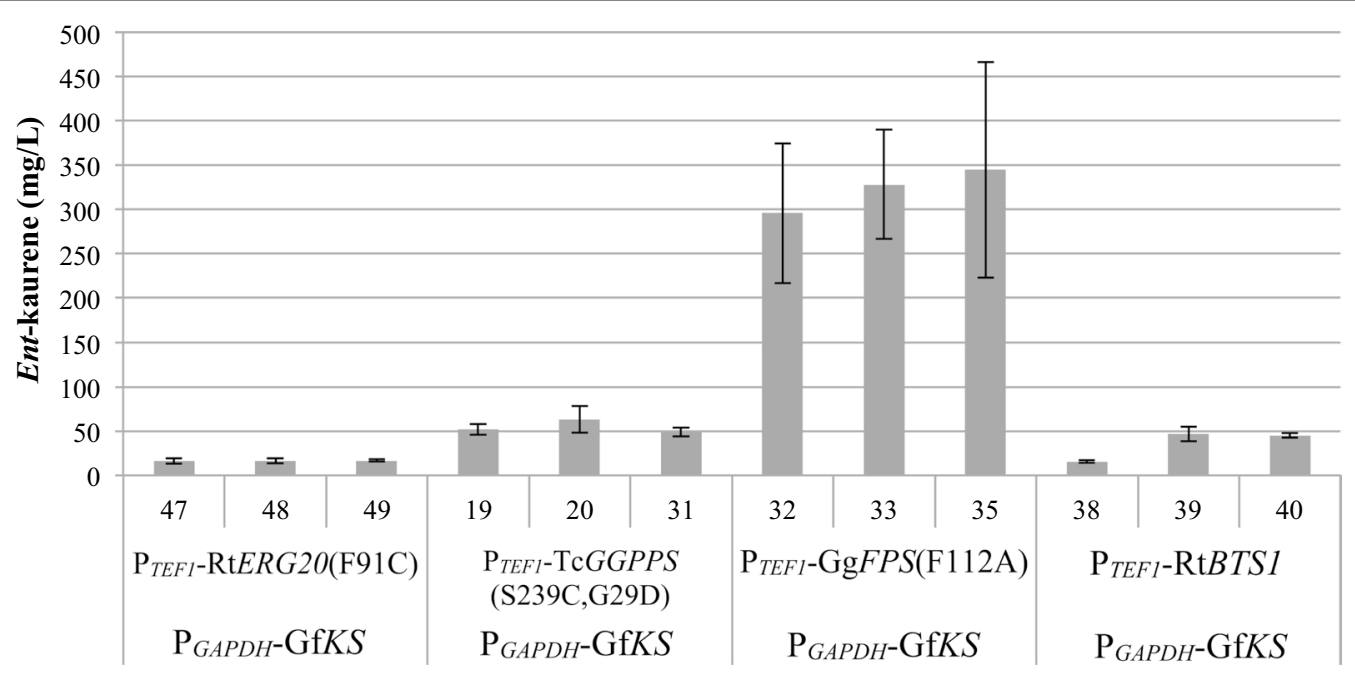

Fig. 4 Co-expression of kaurene synthase from Gibberella fujikuroi (GfKS) and various GGPP synthases in R. toruloides. Strains were constructed by transformation of strain ABFPUB_16 ( $P_{\text {GAPDH }}$-GFKS) with constructs 3 to 6. Ent-kaurene titer at 9 days is shown for the three highest-titer strains for each construct. ( $N=3$, data shown as average \pm standard deviation, from a single experiment in $\mathrm{YPD}_{10}$ medium. ABFPUB identification numbers are listed in Table 1.) 


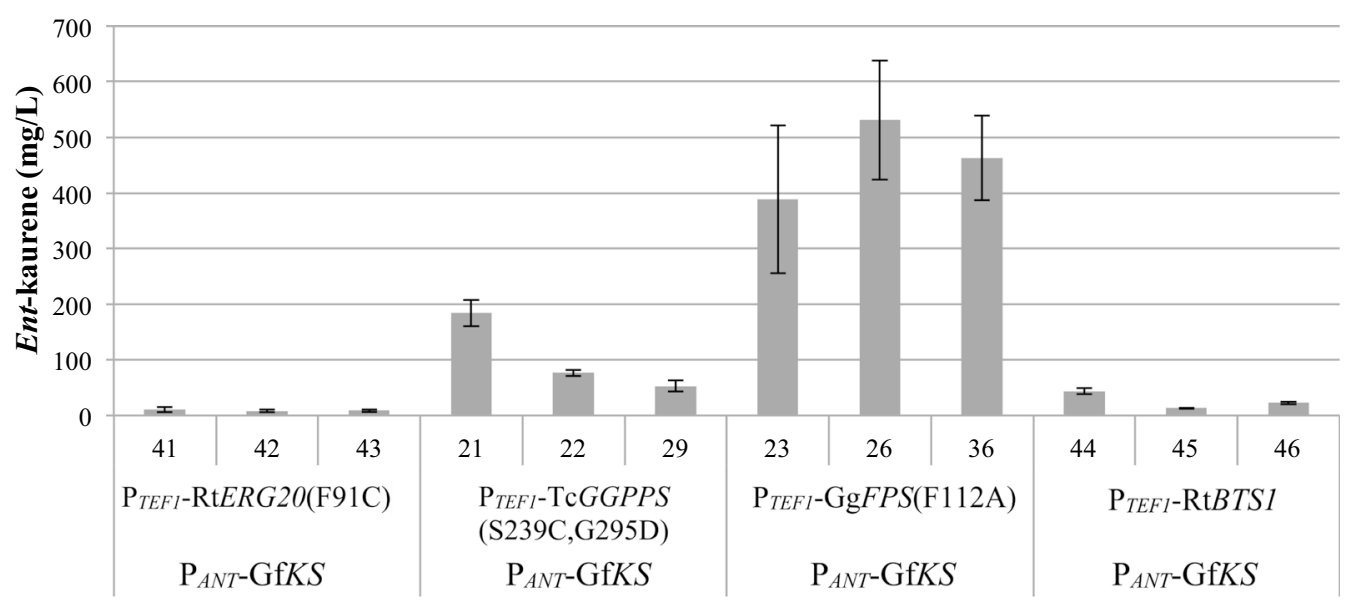

Fig. 5 Co-transformation of kaurene synthase from Gibberella fujikuroi (GfKS) and various GGPP synthases into WT R. toruloides (constructs 7-10, Table 1). Ent-kaurene titer at 9 days is shown for the three highest-titer strains for each construct. $(N=3$, data shown as average \pm standard deviation, from a single experiment in $\mathrm{YPD}_{10}$ medium. ABFPUB identification numbers are listed in Table 1.)
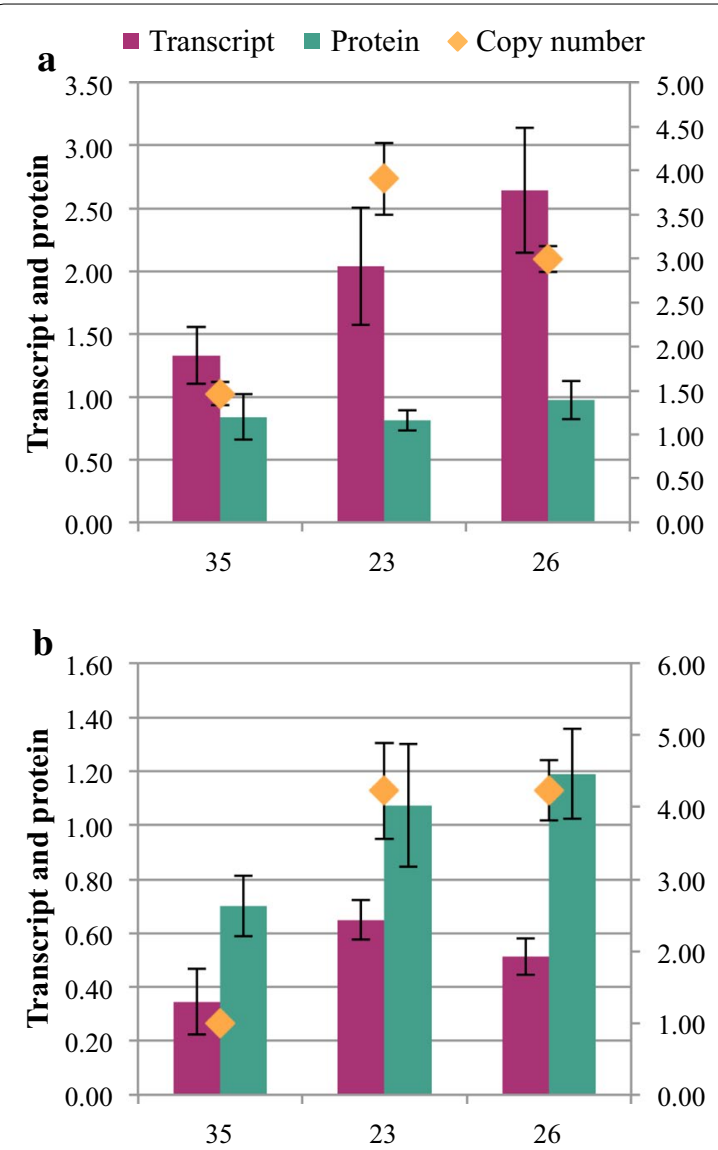

Fig. 6 Average relative abundance of transcript $(\triangle C t)$, protein and copy number of $\mathbf{a}$ GFKS and $\mathbf{b}$ GFFPS(F112A) on day 3. Samples are designated by their ABFPUB identification number. $(N=3$, data shown as average \pm standard deviation, from a single experiment in $\mathrm{YPD}_{10}$ medium.)
One of the advantages of $R$. toruloides as a host is its ability to grow on lignocellulosic hydrolysates and to perform well when cultivated in bioreactors $[26,35]$. To demonstrate production of ent-kaurene from lignocellulose, DMR-EH hydrolysate was prepared from corn stover as described previously [21]. Strain ABFPUB_26 (harboring $\mathrm{P}_{A N T}$ GfKS and $\mathrm{P}_{T E F 1}$-GgFPS(F112A)) was selected for scale-up to a $2 \mathrm{~L}$ bioreactor in a medium composed of $75 \%$ corn stover DMR-EH hydrolysate, supplemented with $10 \mathrm{~g} / \mathrm{L}$ yeast extract. Under these conditions, an ent-kaurene titer of $1.44 \mathrm{~g} / \mathrm{L}$ was achieved (Fig. 7). $\mathrm{OD}_{600}$ and titer increased proportionally, reaching an $\mathrm{OD}_{600}$ of 70 by $281 \mathrm{~h}$; glucose was completely consumed by $161 \mathrm{~h}$ and xylose was fully consumed after 207 h. Strain ABFPUB_26 had produced ent-kaurene at $531 \mathrm{mg} / \mathrm{L}$ when cultivated in $\mathrm{YPD}_{10}$ medium in culture tubes, yet produced 2.7 fold more ent-kaurene when scaled up to a $2 \mathrm{~L}$ bioreactor and 75\% DMR-EH growth medium, which contains only $13 \%$ more sugar $(76 \mathrm{~g} / \mathrm{L}$ glucose and $37 \mathrm{~g} / \mathrm{L}$ xylose) than $\mathrm{YPD}_{10}$. This underscores the compatibility of $R$. toruloides with lignocellulosic feedstocks.

\section{Discussion}

This work applies the Design, Build, Test, and Learn (DBTL) approach to engineer production of the nonnative diterpene ent-kaurene in $R$. toruloides. The ATMT transformation method used in this study promotes random integration of constructs into the $R$. toruloides genome, generating strains that vary in transgene insertion site and copy number. The resulting variation in transgene expression level can be advantageous in that it adds an additional mechanism to adjust transgene 


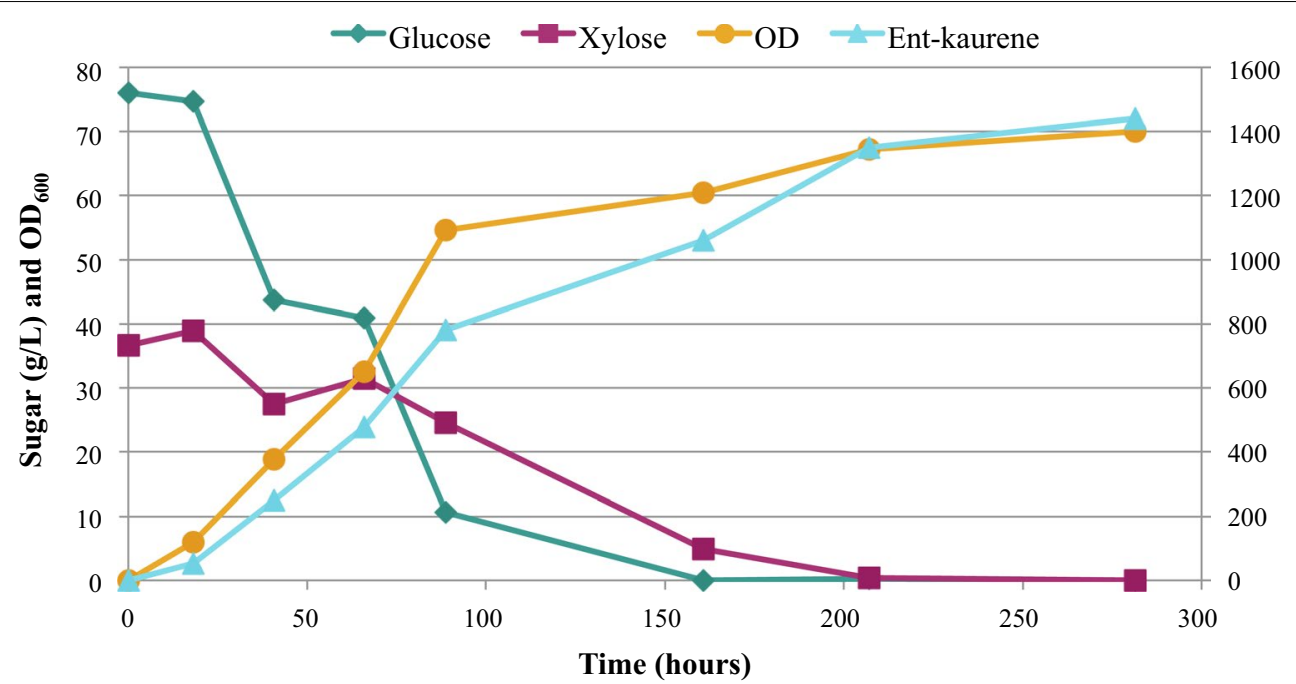

Fig. 7 Sugar concentration, $\mathrm{OD}_{600}$, and ent-kaurene titer data for strain ABFPUB_26 cultivated in a $2 \mathrm{~L}$ bioreactor containing 75\% DMR-EH hydrolysate, supplemented with $10 \mathrm{~g} / \mathrm{L}$ yeast extract

expression in pathway engineering work. However, it also can confound direct comparison of different engineering strategies. Fortunately, this issue can be overcome simply by measuring transgene copy number. For example, a direct comparison between the ANT and GAPDH promoters was made in this study and we observed that $\mathrm{P}_{A N T}$ resulted in stronger GfKS expression than $\mathrm{P}_{G A P D H}$, in agreement with previous data comparing these promoters [32].

Interestingly, unlike previous studies that used these same strong promoters to express a sesquiterpene synthase to produce $100-500 \mathrm{mg} / \mathrm{L}$ of sesquiterpene, simply overexpressing GfKS resulted in almost tenfold lower titers of ent-kaurene [26]. Sesquiterpenes are produced from FPP (the immediate precursor of GGPP), which is a metabolite used for many cellular functions, including biosynthesis of essential sterols such as ergosterol. In contrast, GGPP is used primarily for producing low levels of non-essential carotenoids in $R$. toruloides. Therefore, it's not surprising that this study found evidence that the apparent GGPP metabolite pool is lower than that of FPP. This was substantiated by the significant increase in entkaurene titers observed upon expression of a GGPPS. The variance in ent-kaurene titer in the GGPPS overexpression strains is relatively high, so it's difficult to make conclusive comparisons, but the relative trend in titer suggests that higher levels of GgFPS(F112A) protein promoted higher ent-kaurene titers. These results suggest that the increased GGPPS expression level in the strains generated from a single construct in the final DBTL cycle created a better balance in the pathway, leading to higher flux toward ent-kaurene. Overall, the difference in titer in strains harboring GGPPS constructs was relatively modest, likely indicating that expression of these two terminal enzymes is reaching saturation.

While $\mathrm{g} / \mathrm{L}$ titers of ent-kaurene were achieved by engineering two pathway steps, there are many improvements that can be made to further improve titer, rate and yield (TRY). Additional investigation of the impact of GGPPS expression on the balance between FPP and GGPP could prove fruitful, either from further promoter optimization for both KS and GGPPS expression or by examining other KS and GGPPS orthologs. Beyond these terminal steps in the biosynthetic pathway, optimization of the entire mevalonate pathway will likely lead to improvements in TRY. There are also several broader avenues to explore to facilitate engineering this nascent host. For example, little is known about the impact of integration locus on expression in $R$. toruloides and developing a better understanding of this topic is a high-priority for further investigation. Other engineering tools that would be highly beneficial for pathway optimization include methods to downregulate competing pathways, such as lipid biosynthesis. To that end, the application of RNAi has recently been demonstrated in $R$. toruloides, with downregulation of fatty acid synthases [36]. This tool will be valuable for future studies.

Outside of metabolic engineering approaches, process optimization of cultivation conditions will be important to improve heterologous terpene production in $R$. toruloides. For example, optimization of DMR-EH hydrolysate media to eliminate the use of the yeast extract in favor of a more industrially-relevant nitrogen source such as ammonium sulfate would be ideal. Also, identification 
of other vitamins and minerals that are limiting in DMREH hydrolysates will likely improve TRY. Finally, a deeper exploration of cultivation conditions such as $\mathrm{pH}$, mixing, aeration, and temperature are also needed in this relatively new host organism. Even without these important optimizations, the final titer of $1.44 \mathrm{~g} / \mathrm{L}$ achieved in this study is the highest reported ent-kaurene titer in any microbial cell factory.

\section{Conclusion}

This study builds upon previous work demonstrating the potential of $R$. toruloides as a robust and versatile host for the production of both mono- and sesquiterpenes, and is the first demonstration of the production of a non-native diterpene in this organism. It provides a roadmap for rapid high-titer diterpene production in $R$. toruloides; it is possible that other diterpene synthases could be combined with the GGPPS GgFPS(F112A) in a single expression construct to achieve production of $\mathrm{g} / \mathrm{L}$ quantities of the diterpene. These results, in combination with previous studies on mono- and sesquiterpenes, indicate that $R$. toruloides is an ideal host for the production of a range of different heterologous terpene bioproducts from cheap renewable carbon sources, such as lignocellulosic biomass.

\section{Materials and methods}

\section{Plasmid design and construction}

Plasmids were based on a binary vector for Agrobacterium tumefaciens mediated transformation (ATMT) containing a bacterial expression cassette conferring resistance to kanamycin and $R$. toruloides expression cassettes conferring resistance to nourseothricin (NAT), G418 (KanMX) or hygromycin B (HYG) (Table 1). Coding sequences were optimized for expression in $R$. toruloides, synthesized, and cloned into ATMT plasmid backbones described previously [22, 28], by Genscript (Piscataway, NJ).

\section{Transformation and screening of $R$. toruloides}

Transformation of $R$. toruloides was achieved by ATMT as described previously [22]. Prior to screening, transformants were grown on yeast peptone dextrose (YPD, BD Biosciences, 242820, San Jose, CA) agar containing $300 \mu \mathrm{g} / \mathrm{mL}$ cefotaxime (TCI America, TCI-C2224-25G, Portland, OR) and $300 \mu \mathrm{g} / \mathrm{mL}$ carbenicillin (SigmaAldrich, C1389-5G, St. Louis, MO) to prevent the growth of $A$. tumefaciens. Plates were grown at $30{ }^{\circ} \mathrm{C}$ for three days and single colonies were inoculated into culture tubes containing $5 \mathrm{~mL}$ lysogeny broth (LB, Miller, VWR, J106-500G, Radnor, PA) and cultured overnight at the same temperature with shaking at $200 \mathrm{rpm}$ (Adolf Kühner AG, SBM-X, Birsfelden, Switzerland). Optical density (OD) was determined by measuring absorbance at $600 \mathrm{~nm}\left(\mathrm{OD}_{600}\right)$ with a SpectraMax Plus 384 Microplate Reader (Molecular Devices, PLUS384, San Jose, CA). Cultures were inoculated into $10 \mathrm{~mL}$ YPD at a 1:100 dilution and a 20\% (v/v) dodecane (Sigma-Aldrich, D221104) overlay was added to capture ent-kaurene. Strains were grown at $30{ }^{\circ} \mathrm{C}$ with shaking at $200 \mathrm{rpm}$ for 9-10 days. After an initial round of screening, entkaurene titer of the three highest producing strains was confirmed in triplicate for each construct by growth in $\mathrm{YPD}_{10}$ (YPD containing $100 \mathrm{~g} / \mathrm{L}$ glucose).

\section{Quantification of ent-kaurene}

Following growth of engineered $R$. toruloides cultures, the dodecane phase was sampled and diluted 1:40 into dodecane containing $40 \mathrm{mg} / \mathrm{L}$ pentadecane (SigmaAldrich, 76510), and analyzed by gas chromatographymass spectrometry (GC-MS) using an Agilent 6890 Plus gas chromatograph (Agilent Technologies, G1530A, Santa Clara, CA) connected to an Agilent 5973 Network mass spectrometer (Agilent Technologies, G1099A). $1 \mu \mathrm{L}$ of each sample was injected by a CombiPal autosampler (CTC Analytics, MXY 02-00B, Zwingen, Switzerland). Analytes were separated on a DB-5MS column $(30 \mathrm{~m}$ long, $0.25 \mathrm{~mm}$ internal diameter, $0.25 \mu \mathrm{m}$ film thickness, Agilent Technologies, 122-5532) using the following oven parameters: hold for $0.5 \mathrm{~min}$ at an initial temperature of $100{ }^{\circ} \mathrm{C}$, followed by a temperature ramp of $30^{\circ} \mathrm{C} / \mathrm{min}$ to $250{ }^{\circ} \mathrm{C}$, a ramp of $10{ }^{\circ} \mathrm{C} / \mathrm{min}$ to $270{ }^{\circ} \mathrm{C}$, and a ramp of $30{ }^{\circ} \mathrm{C} / \mathrm{min}$ to $300{ }^{\circ} \mathrm{C}$. The mass spectrometer was operated in selected ion mode, with target ions $(\mathrm{m} / \mathrm{z})$ of 70 , 85,139 and 154. A standard curve was generated by running ent-kaurene standards at concentration range of $5-80 \mu \mathrm{g} / \mathrm{mL}$. Analysis was performed using Enhanced ChemStation (Agilent Technologies, MSD Chemstation E.02.00.493) with ent-kaurene peak areas normalized to peak areas for pentadecane. The ent-kaurene standard was a gift from Dr. Joe Chappell, University of Kentucky, Lexington, $\mathrm{KY}$.

\section{Cultivation at $\mathbf{2} \mathrm{L}$ bioreactor scale}

ABFPUB_26 was selected for growth in lignocellulosic hydrolysate at $2 \mathrm{~L}$ bioreactor scale. Lignocellulosic hydrolysate was prepared from corn stover by deacetylation, mechanical refining and enzymatic hydrolysis, as described previously, and is referred to as DMR-EH hydrolysate [21]. A BIOSTAT $\mathrm{B}^{\circledR}$ fermentation system (Sartorius AG., Goettingen, Germany) was employed in batch mode, using a jacketed $2 \mathrm{~L}$ borosilicate glass vessel (UniVessel ${ }^{\circledR}$, Sartorius AG, Goettingen, Germany) equipped with two 6-blade Rushton impellers, a dissolved oxygen (DO) probe (Hamilton VisiFerm DO 225, Bonaduz, Switzerland), and a $\mathrm{pH}$ probe (Hamilton 
EasyFerm Plus VP 225, Bonaduz, Switzerland). Seed cultures were grown to exponential phase and then used to inoculate $0.75 \mathrm{~L}$ aqueous media (75\% DMR hydrolysate, $10 \mathrm{~g} / \mathrm{L}$ yeast extract, and $30 \mathrm{mg} / \mathrm{L}$ cefotaxime), to which a $20 \%$ organic solvent overlay $(150 \mathrm{~mL}$ dodecane spiked with $200 \mathrm{mg} / \mathrm{L}$ pentadecane as an internal standard) was added to capture ent-kaurene. The culture was grown at $30{ }^{\circ} \mathrm{C}$ with aeration maintained at 0.37 LPM and agitation at $400 \mathrm{rpm}$. After initial adjustment of the growth medium $\mathrm{pH}$ to 5.0 , the $\mathrm{pH}$ was not controlled during growth. Process values were monitored and recorded using the integrated Sartorius data acquisition software (BioPAT MFCS/win). Sugar consumption, OD, and entkaurene production were measured over a period of 12 days. Ent-kaurene was measured by sampling of the dodecane phase, dilution, and analysis by GC-MS.

\section{Determination of glucose and xylose}

Sugars were quantified on a Dionex Ultimate 3000 system UHPLC (Agilent Technologies) using an Aminex HPX-87H column (Bio-Rad, Hercules, CA) and Thermo Scientific $^{\mathrm{TM}}$ RefractoMax 520 Refractive Index Detector (RID) held at $35{ }^{\circ} \mathrm{C}$. Prior to analysis, samples were filtered through $0.45 \mu \mathrm{m}$ filters (VWR Centrifugal Filters) by centrifugation at $3000 \times g$ for $3 \mathrm{~min}$. Samples were run for 26 min using an isocratic $4 \mathrm{mM}$ sulfuric acid mobile phase at $0.6 \mathrm{~mL} \mathrm{~min}{ }^{-1}$ and $65{ }^{\circ} \mathrm{C}$. Glucose, xylose, and arabinose standards were prepared and diluted to create a 7 -point calibration curve ranging from 0.0625 to $4.0 \mathrm{mg} \mathrm{mL}{ }^{-1}$. Standards were run at the beginning and end of each run, and sugar concentrations were calculated using the Chromeleon 7 software package.

\section{Targeted proteomics}

Cultures were grown in culture tubes with $5 \mathrm{~mL} \mathrm{YPD}_{10}$ medium and a dodecane overlay. Time points were collected on day 1 and 3. Protein was extracted using a method based on a previously established protocol [38]. Each cell pellet, corresponding to 6 OD units, was diluted in $200 \mu \mathrm{L}$ of $\mathrm{H}_{2} \mathrm{O}$ and transferred to $2 \mathrm{~mL}$ prefilled Micro-Organism Lysing Mix glass bead tubes and bead beat in a Bead Ruptor Elite bead mill homogenizer (OMNI International, Kennesaw, Georgia) at speed 5.5 for $45 \mathrm{~s}$. After bead beating, the lysate was immediately placed in an ice-block and then spun into a $4 \mathrm{~mL}$ tube at $1,000 \times g$ for $10 \mathrm{~min}$ at $4{ }^{\circ} \mathrm{C}$. To separate the protein, metabolites and lipids, $1 \mathrm{~mL}$ cold $\left(-20{ }^{\circ} \mathrm{C}\right)$ chloroform:methanol mix (prepared 2:1 (v/v)) was pipetted into a chloroform compatible $2 \mathrm{~mL}$ Sorenson MulTI ${ }^{\mathrm{TM}}$ $\mathrm{SafeSeal}^{\mathrm{TM}}$ microcentrifuge tubes (Sorenson bioscience, Salt Lake City, UT) inside an ice-block. The $200 \mu \mathrm{L}$ of sample homogenate was then added to the Sorenson tube at a ratio of 1:5 sample:chloroform mix $(2: 1(\mathrm{v} / \mathrm{v}))$ and vigorously vortexed. The sample was then placed in the ice block for $5 \mathrm{~min}$ and then vortexed for $10 \mathrm{~s}$ followed by centrifugation at $10,000 \times g$ for $10 \mathrm{~min}$ at $4{ }^{\circ} \mathrm{C}$. The upper water-soluble metabolite phase and the lower lipid soluble phase was removed. The remaining protein interlayer had $1 \mathrm{~mL}$ of cold $100 \%$ methanol added to each, vortexed and centrifuged again at $10,000 \times g$ for $10 \mathrm{~min}$ at $4{ }^{\circ} \mathrm{C}$ to pellet the protein. The methanol was then decanted off and the samples were placed in a fume hood to dry for $\sim 10 \mathrm{~min}$. $200 \mu \mathrm{L}$ of an $8 \mathrm{M}$ urea solution was added to the protein pellet and vortexed into solution. A bicinchoninic acid (BCA) assay (Thermo Scientific, Waltham, MA) was performed to determine protein concentration. Following the assay, $10 \mathrm{mM}$ dithiothreitol (DTT) was added to the samples and incubated at $60{ }^{\circ} \mathrm{C}$ for 30 min with constant shaking at $800 \mathrm{rpm}$ followed by the addition of $40 \mathrm{mM}$ iodoacetamide (IAA) with $30 \mathrm{~min}$ of room temperature incubation in the dark. Samples were then diluted eightfold for preparation for digestion with $100 \mathrm{mM} \mathrm{NH}_{4} \mathrm{HCO}_{3}, 1 \mathrm{mM} \mathrm{CaCl}$ and sequencing grade trypsin (USB, Santa Clara, CA) was added to all protein samples at a 1:50 (w/w) trypsin-to-protein ratio for $3 \mathrm{~h}$ at $37^{\circ} \mathrm{C}$. Digested samples were desalted using a 4-probe positive pressure Gilson GX-274 ASPEC $^{\mathrm{TM}}$ system (Gilson Inc., Middleton, WI) with Discovery C18 $50 \mathrm{mg} / 1 \mathrm{~mL}$ solid phase extraction tubes (Supelco, St. Louis, MO), using the following protocol: $3 \mathrm{~mL}$ of methanol was added for conditioning followed by $3 \mathrm{~mL}$ of $0.1 \%$ trifluoroacetic acid (TFA) in $\mathrm{H}_{2} \mathrm{O}$. The samples were then loaded onto each column followed by $4 \mathrm{~mL}$ of $95: 5$ water:acetonitrile, $0.1 \%$ TFA. Samples were eluted with $1 \mathrm{~mL}$ 80:20 acetonitrile:water, 0.1\% TFA. The samples were concentrated down to $\sim 100 \mu \mathrm{L}$ using a Speed Vac and a final BCA was performed to determine the peptide concentration and samples were diluted to $0.20 \mu \mathrm{g} / \mu \mathrm{L}$ with nanopure water for targeted proteomics analysis.

Targeted proteomics was performed via Liquid Chromatography (LC)-Selected Reaction Monitoring (SRM) approach. Five peptides per protein were initially selected based on their SRM suitability scores predicated by CONSeQuence $[39,40]$ software tools. All the peptides were further blasted to ensure their uniqueness to target proteins in the organism. Crude synthetic heavy isotope-labeled (e.g., 13C/15 N on C-terminal lysine and arginine) peptides were purchased from New England Peptide (Gardner, MA). Upon receiving, the crude synthetic heavy peptides were mixed together and diluted with $0.1 \%$ formic acid in $15 \%$ acetonitrile in water to obtain a nominal concentration of $3 \mathrm{pmol} / \mu \mathrm{L}$ for each individual peptide. The heavy peptide mixture stock solution was aliquoted and stored at $-80^{\circ} \mathrm{C}$ until further use.

To develop targeted proteomics assay, all the SRM precursor-fragment ion pairs (i.e., transitions) were 
first analyzed using LC-SRM by spiking heavy peptides in test samples. Three transitions per peptide and three peptides per protein were selected in a final assay based on their LC performance, MS response, transition interferences, endogenous peptide detectability. Collision energies of transitions were obtained using empirical equations provided in Skyline software [41]. The selected peptides, their transitions and collision energy in the final assay are listed in Additional file 1: Table S1.

Crude heavy peptide mixture stock solution was spiked in the $0.20 \mu \mathrm{g} / \mu \mathrm{L}$ peptide samples at a nominal concentration of $37.5 \mathrm{fmol} / \mu \mathrm{L}$ for each peptide. LCSRM analysis utilized a nanoACQUITY UPLC ${ }^{\circledR}$ system (Waters Corporation, Milford, MA) coupled online to a TSQ Altis ${ }^{\mathrm{TM}}$ triple quadrupole mass spectrometer (Thermo Fisher Scientific). The UPLC ${ }^{\circledR}$ system was equipped with an ACQUITY UPLC BEH $1.7 \mu \mathrm{m}$ C18 column $(100 \mu \mathrm{m}$ i.d. $\times 10 \mathrm{~cm})$ and the mobile phases were (A) $0.1 \%$ formic acid in water and (B) $0.1 \%$ formic acid in acetonitrile. $2 \mu \mathrm{L}$ of sample (i.e., $0.4 \mu \mathrm{g}$ of peptides) were loaded onto the column and separated using a 110-min gradient profile as follows (min:flowrate- $\mu \mathrm{L} / \mathrm{min}: \% \mathrm{~B})$ : 0:0.4:1, 6:0.6:1, 7:0.4:1, 9:0.4:6, 40:0.4:13, 70:0.4:22, 80:0.4:40, 85:0.4:95, 91:0.5:95, 92:0.5:95, 93:0.5:50, 94:0.5:95, 95:0.6:1, 98:0.4:1. The LC column is operated with a temperature of $42{ }^{\circ} \mathrm{C}$. The TSQ Altis ${ }^{\mathrm{TM}}$ triple quadrupole mass spectrometer was operated with ion spray voltages of $2100 \pm 100 \mathrm{~V}$ and a capillary inlet temperature of $350{ }^{\circ} \mathrm{C}$. Tube lens voltages were obtained from automatic tuning and calibration without further optimization. Both Q1 and Q3 were set at unit resolution of 0.7 FWHM and Q2 gas pressure was optimized at 1.5 mTorr. The transitions were scanned with a 30 min retention time window and a duty cycle of $0.8 \mathrm{~s}$.

All the LC-SRM data were imported into Skyline software and the peak boundaries were manually inspected to ensure correct peak assignment and peak boundaries. Peak detection and integration were determined based on two criteria: 1 . The same LC retention time and 2. Approximately the same relative peak intensity ratios across multiple transitions between the light peptides and heavy peptide standards. The total peak area ratios of endogenous light peptides and their corresponding heavy isotope-labeled internal standards were then exported from Skyline software as Ratio-toStandard. For each peptide, the total peak area ratios of individual samples were normalized to the average total peak area ratio of all the samples. For each sample, protein abundance was calculated as an average of the normalized total peak area ratios of all three peptides of a protein.

\section{Measurement of transcript levels}

RNA was extracted using the Maxwell 16 AS2000 instrument with a Maxwell RSC Plant RNA Kit (Promega, AS1500, Madison, WI). RNA was quantified with a NanoDrop $^{\mathrm{TM}} 2000$ (Thermo Scientific) and $25 \mathrm{ng}$ was used once linear range had been identified. Relative abundance $(\Delta \mathrm{Ct})$ of transcript levels for GfKS was measured using Superscript IV One-Step RT-PCR System (Thermo Fisher Scientific, 12594100, Waltham, MA) with EvaGreen (Biotium, 31000, Hayward, CA) and a CFX96 Real-Time System C1000 Touch Thermal Cycler (Bio-Rad). Relative abundance was compared with housekeeping genes histone H3 (XP_016270870.1) and actin (XP_016271443.1). Primers are listed in Addition file 2: Table S2.

\section{Copy number quantification}

$1.5 \mathrm{~mL}$ aliquots were sampled from 3-day $R$. toruloides cultures, and centrifuged at $3,000 \times g$ for $5 \mathrm{~min}$ to pellet cells. Genomic DNA was harvested from the cell pellets using the Quick-DNA ${ }^{\mathrm{TM}}$ Fungal/Bacterial Miniprep Kit (Zymo Research) following the manufacturer's instructions. Genomic DNA was quantified by using a NanoDrop $^{\mathrm{TM}} 2000$ (Thermo Scientific). To determine the relative copy number of the introduced transgenes, quantitative PCR was performed using the PowerUp ${ }^{\mathrm{TM}} \mathrm{SYBR}^{\mathrm{TM}}$ Green Master Mix (Thermo Scientific) on a CFX384 Touch Real-Time PCR Detection System (BioRad) using the manufacturer's instructions. Each reaction was set up in triplicate with $1 \mathrm{ng}$ of genomic DNA as template. PCR products (approximately $1 \mathrm{~kb}$ ) spanning the $\mathrm{GPCR}$ amplicons were amplified from genomic DNA for the native sequences and plasmid DNA for the transgenes. These PCR products were gel purified using the Qiaquick gel extraction kit (Qiagen) and used to generate standard curves for each qPCR primer set. Standard curves were used to calculate the copy number of transgenes relative to the native actin and GAPDH (EGU13160.1) genes. Primers are listed in Additional file 2: Table S2.

\section{Supplementary information}

Supplementary information accompanies this paper at https://doi. org/10.1186/s12934-020-1293-8.

Additional file 1: Table S1. Peptide standards used to quantify GkKs and GgFPS(F112A) protein levels in $R$ toruloides and their fragmentation ions.

Additional file 2: Table S2. Primer sequences used for q-PCR and qRT-PCR.

\footnotetext{
Acknowledgements

We would like to thank Sam Coradetti and Joonhoon Kim for providing data analysis help, critical feedback, and resources. We would also like to thank the National Renewable Energy Lab for providing the DMR-EH hydrolysate used in this project. A portion of this research was performed using EMSL (grid.436923.9), a DOE Office of Science User Facility sponsored by the Office
} 
of Biological and Environmental Research and located at Pacific Northwest National Laboratory.

\section{Authors' contributions}

$X Z, J M G, J M S, M B T G$, JK, and GMG conceptualized the project and designed the study. GMG, XZ, JK MBTG, JPP, ERS, YG, NMM, CDN, KEBJ, DMC, and GP conducted experiments, collected data, and performed data analysis. GMG, JK, XZ, JPP, ERS, YG, NMM, CDN, KEBJ, DMC, GP, JKM, DT, JMS and JMG composed the manuscript, gave critical feedback on the manuscript and provided resources. All authors read and approved the final manuscript.

\section{Funding}

Sandia National Laboratories is a multi-mission laboratory managed and operated by National Technology and Engineering Solutions of Sandia, LLC., a wholly owned subsidiary of Honeywell International, Inc., for the U.S. Department of Energy's National Nuclear Security Administration under contract DE-NA0003525. This research was part of the Agile BioFoundry (https://agilebiofoundry.org) supported by the U. S. Department of Energy, Energy Efficiency and Renewable Energy, Bioenergy Technologies Office, through contract DE-AC02-05CH1 1231 between Lawrence Berkeley National Laboratory and the U. S. Department of Energy. The views and opinions of the authors expressed herein do not necessarily state or reflect those of the United States Government or any agency thereof. Neither the United States Government nor any agency thereof, nor any of their employees, makes any warranty, expressed or implied, or assumes any legal liability or responsibility for the accuracy, completeness, or usefulness of any information, apparatus, product, or process disclosed, or represents that its use would not infringe privately owned rights. The Department of Energy will provide public access to these results of federally sponsored research in accordance with the DOE Public Access Plan (http://energy.gov/downloads/doe-public-access-plan).

\section{Availability of data and materials}

All data generated or analyzed during this study are included in this published article.

\section{Ethics approval and consent to participate}

Not applicable.

\section{Consent for publication}

Not applicable.

\section{Competing interests}

The authors declare that they have no competing interests.

\section{Author details \\ 1 Department of Energy, Agile BioFoundry, Emeryville, CA 94608, USA. \\ 2 Department of Biomass Science and Conversion Technology, Sandia National Laboratories, 7011 East Ave, Livermore, CA 94550, USA. ${ }^{3}$ Advanced Biofuels and Bioproducts Process Development Unit, Lawrence Berkeley National Laboratory, Emeryville, CA 94608, USA. ${ }^{4}$ Biological Systems and Engineering Division, Lawrence Berkeley National Laboratory, Berkeley, CA 94720, USA. ${ }^{5}$ Earth and Biological Sciences Directorate, Pacific Northwest National Labora- tory, Richland, WA 99354, USA. ${ }^{6}$ Energy and Environment Directorate, Pacific Northwest National Laboratory, Richland, WA 99354, USA. ${ }^{7}$ QB3-Berkeley, University of California, Berkeley, CA 94704, USA.}

Received: 5 September 2019 Accepted: 23 January 2020

Published online: 05 February 2020

\section{References}

1. Li R, Chou WKW, Himmelberger JA, Litwin KM, Harris GG, Cane DE, et al. Reprogramming the chemodiversity of terpenoid cyclization by remolding the active site contour of epi-isozizaene synthase. Biochemistry. 2014. https://doi.org/10.1021/bi401643u.

2. Sacchettini JC, Poulter CD. Creating isoprenoid diversity. Science. 1997. https://doi.org/10.1126/science.277.5333.1788.

3. Christianson DW. Chemistry. Roots of biosynthetic diversity. Science. 2007. https://doi.org/10.1126/science.1141630.
4. Poulter CD, Rilling HC. The prenyl transfer reaction. Enzymic and mechanistic studies of the 1'-4 coupling reaction in the terpene biosynthetic pathway. Acc Chem Res. 1978. https://doi.org/10.1021/ar50128a004.

5. Poulter CD. Farnesyl diphosphate synthase. A paradigm for understanding structure and function relationships in E-polyprenyl diphosphate synthases. Phytochem Rev. 2006. https://doi.org/10.1007/s1110 1-005-4887-1.

6. Faustino C, Neto Í, Fonte P, Macedo A. Cytotoxicity and chemotherapeutic potential of natural rosin abietane diterpenoids and their synthetic derivatives. Curr Pharm Des. 2018. https://doi.org/10.2174/1381612825 666190112162817.

7. Barros Alencar MVO, Castro E Sousa JM, Rolim HML, Medeiros M das GF, Cerqueira GS, Castro Almeida FR, et al. Diterpenes as lead molecules against neglected tropical diseases. Phytother Res. 2017; https://doi. org/10.1002/ptr.5749.

8. Prieto JM, Silveira D. Natural cytotoxic diterpenoids, a potential source of drug leads for melanoma therapy. Curr Pharm Des. 2018. https://doi. org/10.2174/1381612825666190111143648.

9. Block AK, Vaughan MM, Schmelz EA, Christensen SA. Biosynthesis and function of terpenoid defense compounds in maize (Zea mays). Planta. 2019. https://doi.org/10.1007/s00425-018-2999-2.

10. Xu Y, Charles MT, Luo Z, Mimee B, Tong Z, Véronneau P-Y, et al. UltravioletC priming of strawberry leaves against subsequent Mycosphaerella fragariae infection involves the action of reactive oxygen species, plant hormones, and terpenes. Plant Cell Environ. 2019. https://doi. org/10.1111/pce.13491.

11. Ratnadass A, Wink M. The phorbol ester fraction from Jatropha curcas seed oil: potential and limits for crop protection against insect pests. Int J Mol Sci. 2012. https://doi.org/10.3390/ijms131216157.

12. Yamaguchi S. Gibberellin metabolism and its regulation. Annu Rev Plant Biol. 2008. https://doi.org/10.1146/annurev.arplant.59.032607.092804.

13. Salazar-Cerezo S, Martínez-Montiel N, García-Sánchez J, Pérez-Y-Terrón R, Martínez-Contreras RD. Gibberellin biosynthesis and metabolism: a convergent route for plants, fungi and bacteria. Microbiol Res. 2018. https ://doi.org/10.1016/j.micres.2018.01.010.

14. Camara MC, Vandenberghe LPS, Rodrigues C, de Oliveira J, Faulds C,

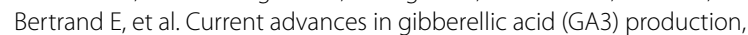
patented technologies and potential applications. Planta. 2018. https:// doi.org/10.1007/s00425-018-2959-x.

15. Takahashi JA, Gomes DC, Lyra FH, Dos Santos GF, Martins LR. The remarkable structural diversity achieved in ent-Kaurane Diterpenes by fungal biotransformations. Molecules. 2014. https://doi.org/10.3390/molecules1 9021856.

16. Du G, Gong H-Y, Feng K-N, Chen Q-Q, Yang Y-L, Fu X-L, et al. Diterpene synthases facilitating production of the kaurane skeleton of eriocalyxin $B$ in the medicinal plant /sodon eriocalyx. Phytochemistry. 2019. https://doi. org/10.1016/j.phytochem.2018.11.015.

17. Hillwig ML, Mann FM, Peters RJ. Diterpenoid biopolymers: new directions for renewable materials engineering. Biopolymers. 2011. https://doi. org/10.1002/bip.21538.

18. Bromann K, Toivari M, Viljanen K, Ruohonen L, Nakari-Setälä T. Engineering Aspergillus nidulans for heterologous ent-kaurene and gamma-terpinene production. Appl Microbiol Biotechnol. 2016. https://doi.org/10.1007/ s00253-016-7517-5.

19. Kong MK, Kang H-J, Kim JH, Oh SH, Lee PC. Metabolic engineering of the Stevia rebaudiana ent-kaurene biosynthetic pathway in recombinant Escherichia coli. J Biotechnol. 2015. https://doi.org/10.1016/j.jbiot ec.2015.09.016

20. Chen X, Shekiro J, Pschorn T, Sabourin M, Tao L, Elander R, et al. A highly efficient dilute alkali deacetylation and mechanical (disc) refining process for the conversion of renewable biomass to lower cost sugars. Biotechnol Biofuels. 2014. https://doi.org/10.1186/1754-6834-7-98.

21. Chen X, Kuhn E, Jennings EW, Nelson R, Tao L, Zhang M, et al. DMR (deacetylation and mechanical refining) processing of corn stover achieves high monomeric sugar concentrations $\left(230 \mathrm{~g} \mathrm{~L}^{-1}\right)$ during enzymatic hydrolysis and high ethanol concentrations (> 10\% v/v) during fermentation without hydrolysate purification or concentration. Energy Environ Sci. 2016. https://doi.org/10.1039/C5EE03718B.

22. Zhuang X, Kilian O, Monroe E, Ito M, Tran-Gymfi MB, Liu F, et al. Monoterpene production by the carotenogenic yeast Rhodosporidium toruloides. Microb Cell Fact. 2019. https://doi.org/10.1186/s12934-019-1099-8. 
23. Singh G, Jawed A, Paul D, Bandyopadhyay KK, Kumari A, Haque S. Concomitant production of lipids and carotenoids in Rhodosporidium toruloides under osmotic stress using response surface methodology. Front Microbiol. 2016. https://doi.org/10.3389/fmicb.2016.01686.

24. Hu C, Zhao X, Zhao J, Wu S, Zhao ZK. Effects of biomass hydrolysis by-products on oleaginous yeast Rhodosporidium toruloides. Bioresour Technol. 2009. https://doi.org/10.1016/j.biortech.2009.04.041.

25. Rodriguez A, Ersig N, Geiselman GM, Seibel K, Simmons BA, Magnuson $\mathrm{JK}$, et al. Conversion of depolymerized sugars and aromatics from engineered feedstocks by two oleaginous red yeasts. Bioresour Technol. 2019. https://doi.org/10.1016/j.biortech.2019.121365.

26. Yaegashi J, Kirby J, Ito M, Sun J, Dutta T, Mirsiaghi M, et al. Rhodosporidium toruloides: a new platform organism for conversion of lignocellulose into terpene biofuels and bioproducts. Biotechnol Biofuels. 2017. https://doi. org/10.1186/s13068-017-0927-5.

27. Li Y, Zhao Z, Bai F. High-density cultivation of oleaginous yeast Rhodosporidium toruloides $Y 4$ in fed-batch culture. Enzyme Microb Technol. 2007. https://doi.org/10.1016/j.enzmictec.2007.02.008.

28. Zhang S, Skerker JM, Rutter CD, Maurer MJ, Arkin AP, Rao CV. Engineering Rhodosporidium toruloides for increased lipid production. Biotechnol Bioeng. 2016. https://doi.org/10.1002/bit.25864.

29. Wehrs M, Gladden JM, Liu Y, Platz L, Prahl J-P, Moon J, et al. Sustainable bioproduction of the blue pigment indigoidine: Expanding the range of heterologous products in $R$. toruloides to include non-ribosomal peptides. Green Chem. 2019. https://doi.org/10.1039/C9GC00920E.

30. Zhuang X. Engineering Novel Terpene Production Platforms in the Yeast Saccharomyces cerevisiae. Doctoral dissertation. University of Kentucky; 2013.

31. Toyomasu T, Kawaide H, Ishizaki A, Shinoda S, Otsuka M, Mitsuhashi W, et al. Cloning of a full-length cDNA encoding ent-kaurene synthase from Gibberella fujikuroi: functional analysis of a bifunctional diterpene cyclase. Biosci Biotechnol Biochem. 2000. https://doi.org/10.1271/bbb.64.660.

32. Nora LC, Wehrs M, Kim J, Cheng J-F, Tarver A, Simmons BA, et al. A toolset of constitutive promoters for metabolic engineering of Rhodosporidium toruloides. BioRxiv. 2019. https://doi.org/10.1101/592774.

33. Tarshis LC, Proteau PJ, Kellogg BA, Sacchettini JC, Poulter CD. Regulation of product chain length by isoprenyl diphosphate synthases. Proc Natl Acad Sci USA. 1996. https://doi.org/10.1073/pnas.93.26.15018.
34. Leonard E, Ajikumar PK, Thayer K, Xiao W-H, Mo JD, Tidor B, et al. Combining metabolic and protein engineering of a terpenoid biosynthetic pathway for overproduction and selectivity control. Proc Natl Acad Sci USA. 2010. https://doi.org/10.1073/pnas.1006138107.

35. Sundstrom E, Yaegashi J, Yan J, Masson F, Papa G, Rodriguez A, et al. Demonstrating a separation-free process coupling ionic liquid pretreatment, saccharification, and fermentation with Rhodosporidium toruloides to produce advanced biofuels. Green Chem. 2018. https://doi.org/10.1039/ C8GC00518D.

36. Liu X, Zhang Y, Liu H, Jiao X, Zhang Q, Zhang S, et al. RNA interference in the oleaginous yeast Rhodosporidium toruloides. FEMS Yeast Res. 2019. https://doi.org/10.1093/femsyr/foz031.

37. Ham TS, Dmytriv Z, Plahar H, Chen J, Hillson NJ, Keasling JD. Design, implementation and practice of JBEI-ICE: an open source biological part registry platform and tools. Nucleic Acids Res. 2012. https://doi. org/10.1093/nar/gks531.

38. Nakayasu ES, Nicora CD, Sims AC, Burnum-Johnson KE, Kim YM, Kyle JE, et al. MPLEx: a robust and universal protocol for single-sample integrative proteomic, metabolomic, and lipidomic analyses. Msystems. 2016. https ://doi.org/10.1128/mSystems.00043-16.

39. Eyers CE, Lawless C, Wedge DC, Lau KW, Gaskell SJ, Hubbard SJ. CONSeQuence: prediction of reference peptides for absolute quantitative proteomics using consensus machine learning approaches. Mol Cell Proteomics. 2011. https://doi.org/10.1074/mcp.M110.003384.

40. Searle BC, Egertson JD, Bollinger JG, Stergachis AB, MacCoss MJ. Using data independent acquisition (DIA) to model high-responding peptides for targeted proteomics experiments. Mol Cell Proteomics. 2015. https:// doi.org/10.1074/mcp.M115.051300.

41. MacLean B, Tomazela DM, Shulman N, Chambers M, Finney GL, Frewen $B$, et al. Skyline: an open source document editor for creating and analyzing targeted proteomics experiments. Bioinformatics. 2010. https://doi. org/10.1093/bioinformatics/btq054.

\section{Publisher's Note}

Springer Nature remains neutral with regard to jurisdictional claims in published maps and institutional affiliations.
Ready to submit your research? Choose BMC and benefit from:

- fast, convenient online submission

- thorough peer review by experienced researchers in your field

- rapid publication on acceptance

- support for research data, including large and complex data types

- gold Open Access which fosters wider collaboration and increased citations

- maximum visibility for your research: over 100M website views per year

At BMC, research is always in progress.

Learn more biomedcentral.com/submissions 